\title{
Dual-modality imaging demonstrates the enhanced antitumoral effect of herpes simplex virus-thymidine kinase/ganciclovir plus gemcitabine combination therapy on cholangiocarcinoma
}

\author{
JIANFENG WANG ${ }^{1}$, ANG LI $^{2}$, MEI JIN ${ }^{3}$, FAN ZHANG ${ }^{4}$ and XIAOLING $\mathrm{LI}^{4}$ \\ ${ }^{1}$ Department of Interventional Radiology, Beijing Chaoyang Hospital, Beijing 100020; \\ ${ }^{2}$ Department of Biostatistics, Public Health School, Harbin Medical University, Harbin, Heilongjiang 150081; \\ ${ }^{3}$ Department of Radiology, First Hospital of Qiqihar, Qiqihar, Heilongjiang 161006; ${ }^{4}$ Department of Radiology, \\ First Hospital of Heilongjiang University of Chinese Medicine, Harbin, Heilongjiang 150040, P.R. China
}

Received November 25, 2014; Accepted January 14, 2016

DOI: $10.3892 /$ etm.2016.3294

\begin{abstract}
Herpes simplex virus-thymidine kinase/ganciclovir (HSV-TK/GCV) therapy is one of the most promising therapeutic strategies for the treatment of cholangiocarcinoma, which is the second most common hepatobiliary cancer. The aim of the present study was to evaluate the enhanced therapeutic effects of HSV-TK/GCV with gemcitabine on cholangiocarcinoma. QBC939 cholangiocarcinoma cells and mouse models of cholangiocarcinoma (established via tumor xenografts) received one of the following treatments: i) Gemcitabine therapy ( $3 \mu \mathrm{g} / \mathrm{ml})$; ii) HSV-TK/GCV monotherapy; iii) HSV-TK/GCV + gemcitabine; and iv) control group, treated with phosphate-buffered saline. Cell proliferation was quantified using MTT assay and post-treatment tumor alterations were monitored using ultrasound imaging and optical imaging. For the in vitro experiments, the MTT assays demonstrated that the relative cell viabilities in the gene therapy, gemcitabine and gemcitabine + gene groups were $70.37 \pm 9.07,52.64 \pm 8.28$ and $34.21 \pm 6.63 \%$, respectively. For the in vivo experiments, optical imaging indicated significantly decreased optical signals in the combination therapy group, as compared with the gemcitabine and gemcitabine + gene groups ( $1.68 \pm 0.74$ vs. $2.27 \pm 0.58$ and $2.87 \pm 0.82$, respectively; $\mathrm{P}<0.05$ ). As demonstrated by ultrasound imaging, reduced tumor volumes were detected in the combination therapy group,
\end{abstract}

Correspondence to: Dr Xiaoling Li, Department of Radiology, First Hospital of Heilongjiang University of Chinese Medicine, 26 Heping Road, Harbin, Heilongjiang 150040, P.R. China E-mail: xiaolinglihrb@126.com

Abbreviations: HSV-TK, herpes simplex virus-thymidine kinase; GCV, ganciclovir; PBS, phosphate-buffered saline; RT-qPCR, reverse transcription-quantitative polymerase chain reaction; ROI, region of interest; RSI, relative signal intensities

Key words: cholangiocarcinoma, herpes simplex virus-thymidine kinase/ganciclovir, gemcitabine, optical imaging as compared with the three control groups $(114.32 \pm 17.17$ vs. $159 \pm 23.74,201.63 \pm 19.26$ and $298.23 \pm 36.1 \mathrm{~mm}^{3}$, respectively; $\mathrm{P}<0.05)$. The results of the present study demonstrated that gemcitabine enhances the antitumoral effects of HSV-TK/GCV on cholangiocarcinoma, which may provide a novel therapeutic strategy for the management and treatment of cholangiocarcinoma using gemcitabine and gene therapy.

\section{Introduction}

Cholangiocarcinoma, which is the second most common hepatobiliary cancer, is difficult to diagnose in the early stages, particularly in extrahepatic cholangiocarcinoma (1). The majority of patients with cholangiocarcinoma present with jaundice and an advanced stage tumor, resulting in low resectability and curability, and a poor long-term survival rate $(1,2)$. Gemcitabine has been increasingly used to treat patients with cholangiocarcinoma $(3,4)$, and a chemotherapy regimen of gemcitabine is commonly used for inoperable cholangiocarcinoma; however, the efficacy is not satisfactory (5).

Gene therapy is a promising therapeutic strategy for treatment of patients with cholangiocarcinoma $(6,7)$, and herpes simplex virus-thymidine kinase/ganciclovir (HSV-TK/GCV) suicide gene therapy is considered one of the most promising therapeutic strategies for the treatment of cholangiocarcinoma $(8,9)$. Expression of the HSV-TK gene induces the production of thymidine kinase, which metabolizes GCV to GCV monophosphate, and cellular kinases subsequently convert monophosphorylated GCV into its diphosphate and triphosphate forms. GCV triphosphate is incorporated into DNA during cell division, resulting in single-strand DNA breaks and the inhibition of DNA polymerase, which induces DNA chain termination (10). These effects induce apoptotic mechanisms (11), thus producing an antitumor effect. A previous study has suggested that gemcitabine may improve the efficacy of HSV-TK/GCV gene therapies (12). Therefore, in the current study, the efficacy of HSV-TK was evaluated alone, and in combination with gemcitabine, in QBC929 cells (selected and derived from a patient with cholangiocarcinoma) and a mouse model of cholangiocarcinoma. The aim 
of the study was to determine whether HSV-TK/GCV plus gemcitabine may be essential for anti-tumor growth, and may therefore prove to be a novel therapeutic method to enhance the efficacy of cholangicarcinoma chemotherapy.

\section{Materials and methods}

Cell culture and the efficiency of gene transfer. QBC939 human cholangiocarcinoma cell line was obtained from Suer Biological Inc. (Shanghai, China). Cells were maintained in RPMI 1640 medium supplemented with $10 \%$ fetal bovine serum (Gibco; Thermo Fisher Scientific, Inc., Waltham, MA, USA), $100 \mathrm{U} / \mathrm{ml}$ penicillin and $100 \mu \mathrm{g} / \mathrm{ml}$ streptomycin (Shanghai Institute Hui Biotechnology Co., Ltd., Shanghai, China) in a humidified incubator (18 M; Sanyo Electric Co., Ltd., Osaka, Japan) containing $5 \% \mathrm{CO}_{2}$ at $37^{\circ} \mathrm{C}$.

A total of $5 \times 10^{4}$ cells/well were plated in 24-well plates and incubated overnight. Cells were transfected with lipofectamine 2000 (Sigma-Aldrich, St. Louis, MO, USA) and HSV/luciferase/lentivirus supernatant (provided by Department of Molecular Biology, Heilongjiang University of Chinese Medicine, Harbin, China) at a dosage of $10^{6} \mathrm{IU} / \mathrm{cell}$ in the presence of $8 \mu \mathrm{g} / \mathrm{ml}$ polybrene. The viral supernatant was removed from the wells after 6-8 h, and the cells were re-infected with fresh supernatant containing polybrene. The following day, the viral supernatant was removed and the appropriate complete growth medium (Gibco; Thermo Fisher Scientific, Inc.) was added to the cells prior to incubation at $37^{\circ} \mathrm{C}$ in an atmosphere containing $5 \% \mathrm{CO}_{2}$ for $72 \mathrm{~h}$. Following incubation, the cells were subcultured into 100-mm dishes and treated with $200 \mu \mathrm{g} / \mathrm{ml}$ G418 (Real-Times Biotechnology Co., Ltd., Beijing, China) for 2 weeks in order to select for stable cell lines. Positive clones were selected and expanded to establish the cell lines. Transfected cells stably expressing HSV-TK, as verified by reverse transcription-quantitative polymerase chain reaction (RT-qPCR), were named QBC939/HSV.

Cell proliferation assay. QBC939/HSV cells (5x10³ cells/well) were cultured in 96-well plates. Media was changed on the second day following plating and the cells were exposed to one of four following treatment options: i) Gemcitabine ( $3 \mu \mathrm{g} / \mathrm{ml}$; Eli Lilly \& Co., Indianapolis, IN, USA) only for 24 h, ii) HSV-TK and GCV (50 $\mu \mathrm{g} / \mathrm{ml}$; Wuhan Hiteck Biological Pharma Co., Ltd., Wuhan, China) only for $72 \mathrm{~h}$, iii) HSV-T plus gemcitabine ( $3 \mu \mathrm{g} / \mathrm{ml}$ ) for $24 \mathrm{~h}$ followed by media removal and replacement with media containing $50 \mu \mathrm{gml} \mathrm{GCV}$ for $72 \mathrm{~h}$, and iv) phosphate-buffered saline (PBS) control group. GCV and gemcitabine were dissolved in sterile distilled water and diluted in culture medium immediately prior to use. Gemcitabine and GCV dosages were selected according to previous studies $(13,14)$.

Cell proliferation was evaluated by MTT assay (Beijing CellChip Biotechnology Co., Ltd., Beijing, China). Briefly, $1 \mathrm{mg} / \mathrm{ml}$ MTT was added to the wells and incubated in an atmosphere containing $5 \% \mathrm{CO}_{2}$ for $4 \mathrm{~h}$. Absorbance was measured at $490 \mathrm{~nm}$ using a microplate reader (FLx800; BioTek, Winooski, VT, USA). Relative cell proliferation rates of the various groups were evaluated using the equation $\mathrm{A}_{\text {treated }} / \mathrm{A}_{\text {control }}$, where $\mathrm{A}$ is the absorbance. All experiments were repeated six times for each cell group.
$R T$ - $q P C R$. Total RNA was extracted from QBC939/HSV cells using an AllPrep DNA/RNA Mini kit (Qiagen, Inc., Valencia, CA, USA). RNA purification was performed using an RNeasy Kit (Qiagen, Inc.). RT-qPCR was performed using an Mx3005P ${ }^{\text {тм }}$ RT-qPCR system (Stratagene; Agilent Technologies, Inc., Santa Clara, CA, USA). Reverse transcription was performed at $42^{\circ} \mathrm{C}$ for $1 \mathrm{~h}$. Super Taq Polymerase (Applied Biosystems; Thermo Fisher Scientific, Inc.) was used in the PCR reaction. RT-qPCR amplification mixtures $(25 \mu \mathrm{l})$ contained $25 \mathrm{ng}$ template cDNA, 2x SYBR Green I Master Mix buffer (12.5 $\mu \mathrm{l}$; Applied Biosystems; Thermo Fisher Scientific, Inc.) and $300 \mathrm{nM}$ forward and reverse primers. Reactions were run on an ABI PRISM 5700 Sequence Detector (Applied Biosystems; Thermo Fisher Scientific, Inc.). The thermal cycling conditions were as follows: Activation for $10 \mathrm{~min}$ at $95^{\circ} \mathrm{C}$, denaturation for 40 cycles at $95^{\circ} \mathrm{C}$ for $15 \mathrm{sec}$, and annealing/extension at $60^{\circ} \mathrm{C}$ for $60 \mathrm{sec}$. The following primers were used: HSV-TK, forward 5'-GGTGATGACCTC TGCCCAGAT-3', and reverse 5'-TGTGAGGAGCCAGAA CAGCAT-3'; and a human control GAPDH primer set from the RT-qPCR kit. PCR cycling was performed as follows: Initial denaturation at $50^{\circ} \mathrm{C}$ for 2 min followed by 35 cycles of $94^{\circ} \mathrm{C}$ for $30 \mathrm{sec}, 54^{\circ} \mathrm{C}$ for $30 \mathrm{sec}$ and $72^{\circ} \mathrm{C}$ for $30 \mathrm{sec}$, and final elongation at $72^{\circ} \mathrm{C}$ for $5 \mathrm{~min}$. Each assay included (in duplicate): A standard curve of four serial dilution points of cDNA (ranging, $50 \mathrm{ng}-50 \mathrm{pg}$ ), a no-template control, a no RT control and $25 \mathrm{ng}$ of each test cDNA. PCR products were analyzed using 3\% agarose gel electrophoresis stained with ethidium bromide using Applied Biosystems Sequence Detection Software version 1.3 (Applied Biosystems; Thermo Fisher Scientific, Inc.).

Animal model and therapy. The animal protocol of the present study was approved by the Institutional Animal Care and Use Committee. At the end of the experiments, mice were sacrificed by asphyxiation with $\mathrm{CO}_{2}(99.9 \%)$. Cholangiocarcinoma tumor xenografts were established in 24 mice (age, 4-6 weeks) by subcutaneously inoculating the left back of each mouse with QBC939 cells $\left(5 \times 10^{6}\right.$ cells/well). Once the tumor size was $8-10 \mathrm{~mm}$ in diameter, the mice were randomly divided into four groups: i) Gemcitabine group, intratumoral injection of $100 \mu \mathrm{l}$ phosphate-buffered saline (PBS), followed by intraperitoneal injection of $50 \mathrm{mg} / \mathrm{kg}$ gemcitabine; ii) gene, intratumoral injection of HSV-TK gene $\left(10^{8}\right)$ in $100 \mu \mathrm{l} \mathrm{PBS}$, followed by intraperitoneal injection of $50 \mathrm{mg} / \mathrm{kg} \mathrm{GCV}$; iii) gemcitabine + gene, intratumoral injection of HSV-TK gene $\left(10^{8}\right)$ in $100 \mu \mathrm{l} \mathrm{PBS}$, followed by intraperitoneal injection of $50 \mathrm{mg} / \mathrm{kg} \mathrm{GCV}$ and $50 \mathrm{mg} / \mathrm{kg}$ gemcitabine; and iv) control, intratumoral injection of $100 \mu \mathrm{l}$ PBS, followed by intraperitoneal injection of PBS. Gemcitabine was injected once, and GCV was injected for 14 days. Gemcitabine and GCV dosages were selected according previous studies $(15,16)$. The general conditions of the mice were monitored daily.

Imaging studies. Mice were anesthetized with 5\% isoflurane gas (Shandong Keyuan Pharmaceutical Co., Jinan, China) and maintained with $2 \%$ isoflurane in a supine position in order to perform optical and ultrasound (US) imaging. Images were captured at day 1 prior to treatment and at days 4, 7, 10 and 14 following treatment. 
Optical imaging was performed using an Fx Pro molecular imaging system (Bruker Bio Spin Corporation, Billerica, MA, USA). Intraperitoneal injection of $300 \mathrm{mg} / \mathrm{kg}$ d-luciferin (Shanghai Yeasen Biotechnology Co., Ltd., Shanghai, China) was administered and images were captured at $20 \mathrm{~min}$ (binning, 4x4). Exposure time was adjusted for each image in order to ensure that the acquired images were presented in the same scale. X-ray was exposed for $2 \mathrm{~min}$. The region of interest (ROI) was drawn using the imaging software (Fx Pro; Bruker Bio Spin Corporation), and the photon flux (photon/s) was subsequently measured. An untreated site on each image was used to normalize the signals against background noise.

Tumor sizes were measured using a Mindray DC-T6 US machine (Mindary Medical International, Ltd., Beijing China). The tumor mass was coated in warmed $\left(37^{\circ} \mathrm{C}\right)$ Aquasonic ultrasound gel (Parker Laboratories, Inc., Fairfield, NJ, USA) and centered on the imaging plane. The two longest perpendicular axes were positioned at the $\mathrm{X}$ and $\mathrm{Y}$ planes, and the depth axes, defined as the $\mathrm{Z}$ of each tumor mass, were measured. The volume of each tumor mass was then calculated according to following equation: Volume $=\mathrm{X} \times \mathrm{Y} \times \mathrm{Z} \times \pi / 6$.

Histological conformation. Tumor masses of the mice were harvested at day 14 following treatment. Tumor tissues were embedded in optical cutting temperature compound (Sakura Finetek USA, Inc., Torrance, CA, USA), frozen in liquid nitrogen and maintained at $-80^{\circ} \mathrm{C}$. Tumor tissues were then cryosectioned into $10-\mu \mathrm{m}$ sections for apoptosis, and hematoxylin and eosin (Boster Biotechnology Co., Ltd., Wuhan, China) staining. Level of apoptosis was determined via a terminal deoxynucleotidyl transferase dUTP nick end-labeling assay using a TACS XL Blue Label kit (Trevigen, Gaithersburg, MD, USA) according to the manufacturer's protocol. For each slide, images from random six fields were captured using an Olympus digital camera (IX53; Olympus Corporation, Tokyo, Japan). Apoptosis results were analyzed using the apoptotic index, which was defined as the number of apoptotic cells/total number of cells in each field.

Statistical analysis. SPSS version 19.0 (IMB SPSS, Amronk, NY, USA) was used to perform all data analysis. Data were presented as the mean \pm standard deviation. A non-parametric Mann-Whitney U test was used to compare the relative proliferation rates between the different in vitro cell groups and the relative signal intensities (RSI) of fluorescence between the different in vivo mice groups. RSI values were normalized using the following equation: $\mathrm{RSI}=\mathrm{SI} \mathrm{Dn} / \mathrm{SI} \mathrm{D} 0$, where SI is the signal intensity, Dn represents the days after treatment and D0 is the day prior to treatment. Between-in vivo group differences in the tumor volumes were analyzed using Student's t-test. $\mathrm{P} \leq 0.05$ was considered to indicate a statistically significant difference.

\section{Results}

Administration of gemcitabine + HSV-TK significantly decreases cell proliferation in vitro. In vitro, the relative viability of cells in the gene treatment, gemcitabine and gemcitabine + gene groups $(70.37 \pm 9.07,52.64 \pm 8.28$ and $34.21 \pm 6.63 \%$, respectively were significantly decreased, as

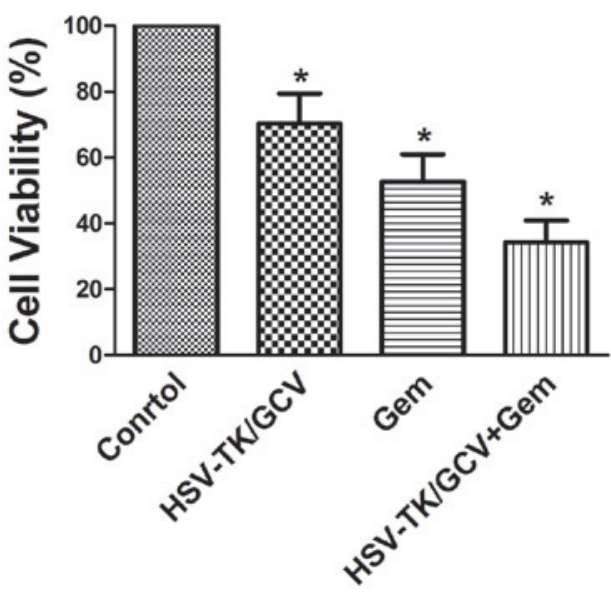

Figure 1. In vitro experiments investigating cell viability demonstrated that administration of Gem + HSV-TK resulted in significantly decreased cell proliferation, as compared with the other groups. ${ }^{*} \mathrm{P}<0.05$ vs. the control group. HSV-TK, herpes simplex virus-thymidine kinase; Gem, gemcitabine.

compared with the control group ( $\mathrm{P}<0.05 ;$ Fig. 1). Furthermore, cell viability was lowest in the combined treatment group and was significantly reduced, as compared with the gene treatment and gemcitabine monotherapy groups $(\mathrm{P}<0.05)$. Relative cell viability values were significantly lower in the cell group receiving gemcitabine, as compared with the HSV-TK + GCV group (52.64 \pm 8.28 vs. $70.37 \pm 9.07 \%$; $\mathrm{P}<0.05)$. HSV-TK mRNA expression levels in the transduced QBC939 cells were assessed using RT-qPCR and agarose gel electrophoresis was used to detect the HSV-TK mRNA 237-bp product.

Administration of gemcitabine $+H S V-T K$ significantly decreases tumor signals in a mouse model of subcutaneous cholangiocarcinoma. All mice survived the in vivo experiments performed in the present study. Fig. 2 shows the optical images captured of the various groups. Follow-up optical imaging on day 14 after treatment demonstrated significantly decreased optical signals in the gemcitabine + gene group, as compared with the other three groups ( $1.68 \pm 0.74$ vs. $2.27 \pm 0.58$, $2.87 \pm 0.82$ and $3.79 \pm 0.72$, respectively; $\mathrm{P}<0.05$ ).

Gemcitabine + HSV-TK combination therapy significantly reduces tumor volume in a mouse model of subcutaneous cholangiocarcinoma. The mean volumes of the tumors prior to treatment in the control, gemcitabine-only, HSV-TK + GCV and combination therapy groups were $96.71 \pm 11.12$, $87.68 \pm 12.27,98.39 \pm 10.20$ and $95.32 \pm 9.81 \mathrm{~mm}^{3}$, respectively. Although gene or gemcitabine monotherapy significantly inhibited tumor growth $(1.68 \pm 0.74$ and $2.27 \pm 0.58$, respectively; $\mathrm{P}<0.05)$, combination therapy induced greater inhibition of tumor development and the most significant delay in tumor growth $(2.87 \pm 0.82 ; \mathrm{P}<0.05)$, as determined by tumor volume on days 7, 10 and 14 following initial treatment (Fig. 3). Tumor volume on day 14 following treatment was significantly reduced in the gemcitabine + HSV-TK group, as compared with the gemcitabine monotherapy group (114.32 \pm 17.17 vs. $159 \pm 23.74 ; \mathrm{P}<0.05)$, gene only $(114.32 \pm 17.17$ vs. $201.63 \pm 19.26$; $\mathrm{P}<0.05)$ and the control group $(114.32 \pm 17.17$ vs. $298.23 \pm 46.35$; $\mathrm{P}<0.01)$ (Figs. 3 and 4). Furthermore, the apoptosis index was 


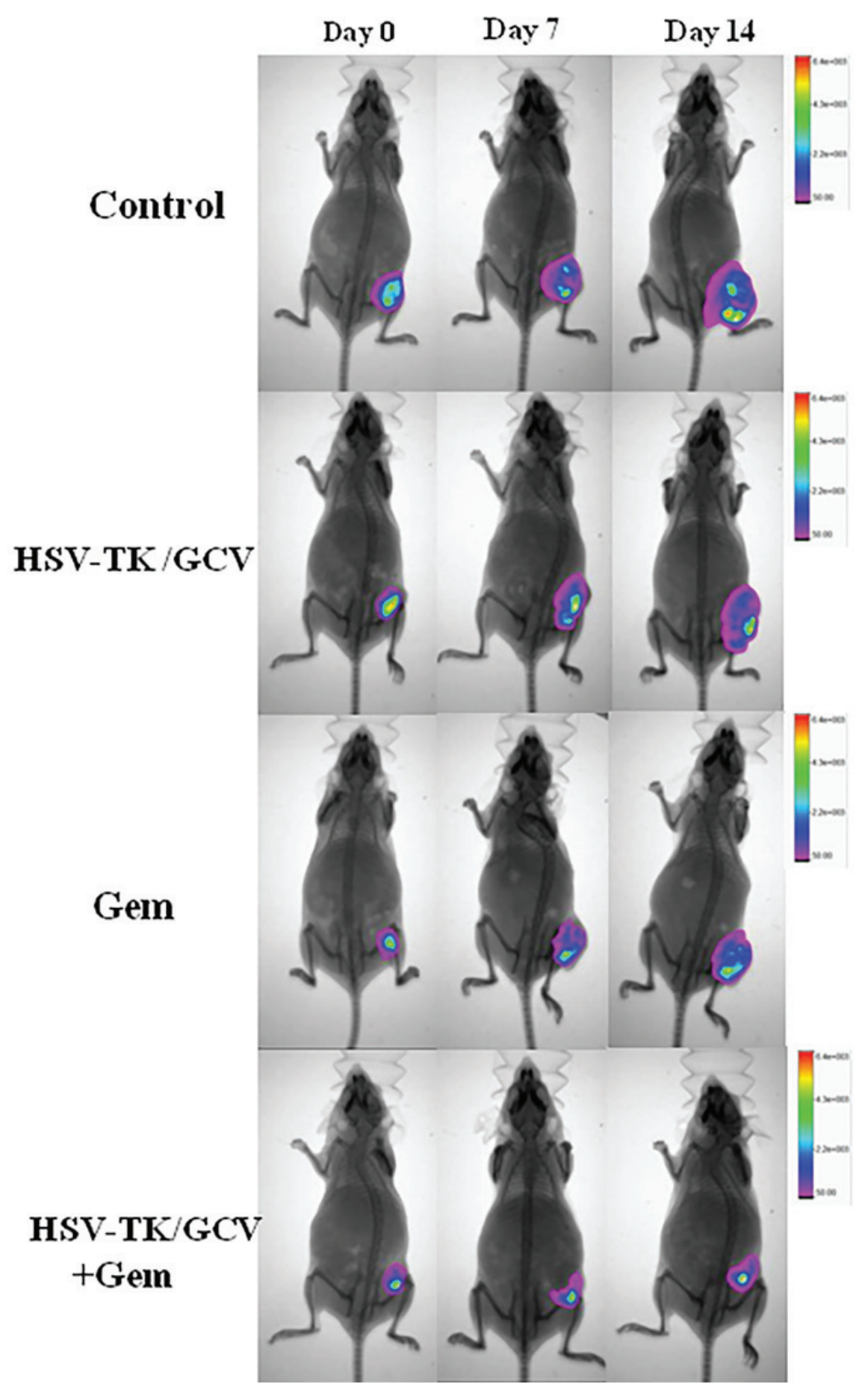

Figure 2. As demonstrated by in vivo optical imaging, decreased tumor signals were detected on day 14 following treatment in the Gem + HSV-TK group, as compared with the other groups. HSV-TK, herpes simplex virus-thymidine kinase; Gem, gemcitabine.

significantly increased in the gemcitabine + HSV-TK group, as compared with the gemcitabine $(41 \pm 8$ vs. $24 \pm 6 \%$; $\mathrm{P}<0.05)$, HSV-TK + GCV ( $41 \pm 8$ vs. $16 \pm 5 \% ; \mathrm{P}<0.05)$ and control $(41 \pm 8$ vs. $4 \pm 1 \% ; \mathrm{P}<0.05)$ groups. These results indicated the enhanced cell killing effects of gemcitabine + HSV-TK combination therapy.

\section{Discussion}

Cholangiocarcinoma is one of the most difficult malignancies to treat and mortality rates remain very high. The most effective treatment for cholangiocarcinoma is curative surgical resection of the primary tumor; however, this procedure is complex and is dependent on the site and extent of the tumor. Furthermore, once diagnosed, the majority of patients are already at the late stages of disease and are no longer candidates for surgery (2). Therefore, the clinical management of cholangiocarcinoma remains a major concern (17).

Gemcitabine is the only chemotherapeutic agent that has been demonstrated to have a significant impact on either survival or disease-related symptoms in patients with pancreatic carcinoma (18). Biliary tract cancers are considered 


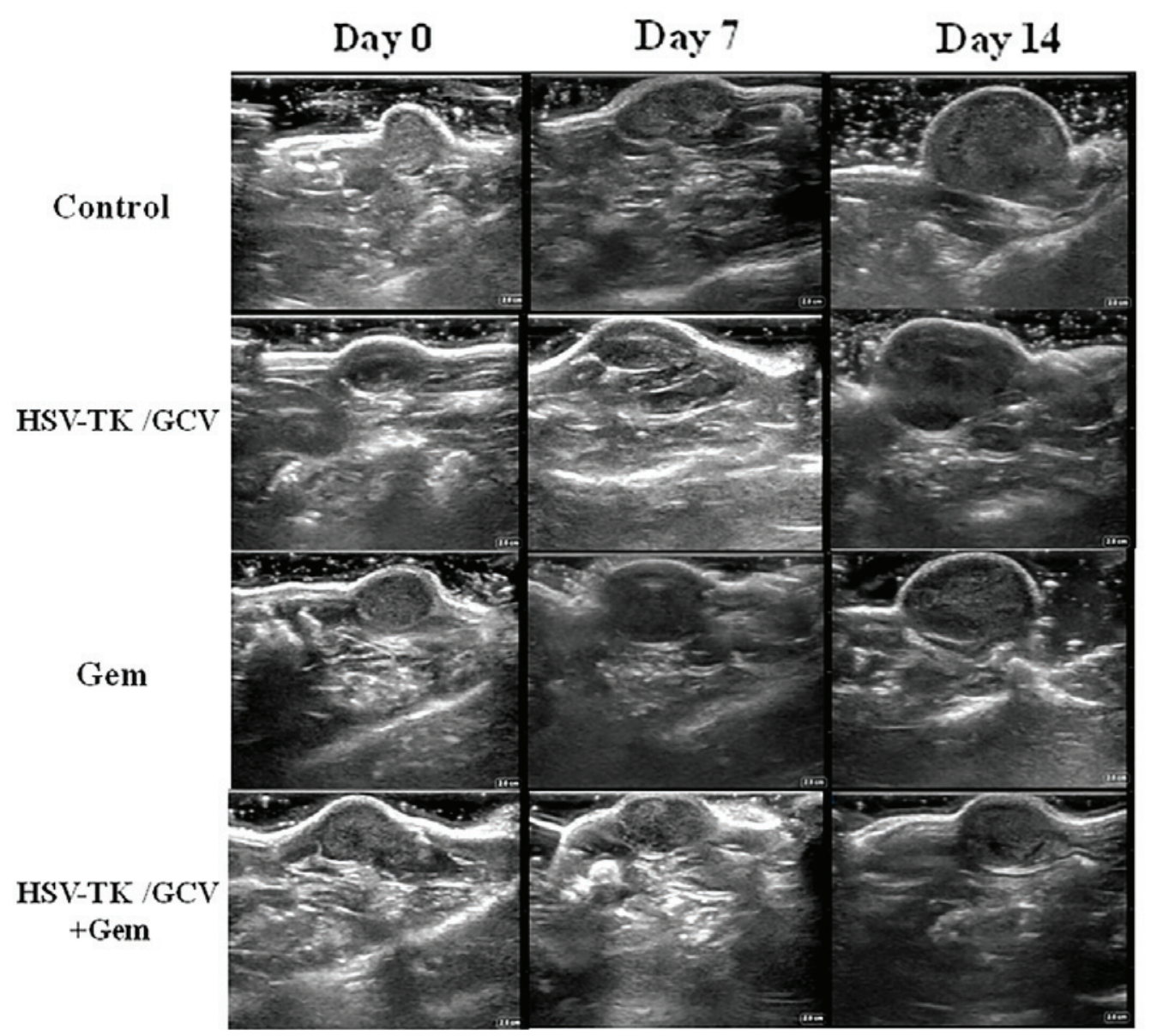

Figure 3. Ultrasound imaging demonstrated the relative tumor volume of the tumors in the Gem + HSV-TK group decreased by day 14 following treatment, as compared with the other groups. HSV-TK, herpes simplex virus-thymidine kinase; Gem, gemcitabine.

similar to pancreatic cancer in aggressiveness and sensitivity to chemotherapy (19). Due to the lack of effective treatment options for cholangiocarcinoma, gemcitabine-based chemotherapy for advanced cholangiocarcinoma has been widely used in the past decade and is accepted as the standard chemotherapeutic agent for the treatment of cholangiocarcinoma $(4,20)$. Gemcitabine, either alone or in combination with other therapeutic agents, including fluoropyrimidines or cisplatin, has been demonstrated to have positive activity and response in treating advanced cholangiocarcinoma. Response rates of patients administered single-agent gemcitabine have varied between $0-30 \%$, with median overall survival times ranging between 5-14 months (17). However, the outcome of gemcitabine-based chemotherapy remains poor due to the high resistance of cancer cells to gemcitabine; therefore, novel therapeutic approaches are necessary (5).

Tang et al (21) established a rat model of bladder tumors. The anaerobic Bifidobacterium infantis-mediated HSV-TK was injected into tumor-bearing rats via the tail vein, followed by intraperitoneal injection of GCV. The results demonstrated that bladder tumor burdens were significantly lower in the rats treated with HSV-TK + GCV compared with the control group $(\mathrm{P}<0.05)$. While various degrees of apoptosis of the tumor cells were detected in all groups using an in situ TUNEL assay, apoptosis was mostly notable in the Bifidobacterium infantis-mediated HSV-TK + GCV treatment

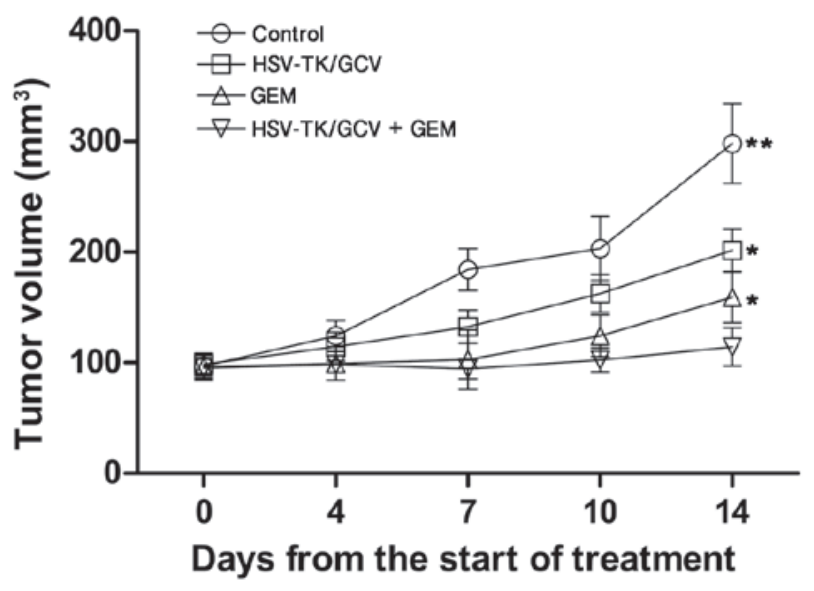

Figure 4. Tumor volumes in a nude mouse model of subcutaneous cholangiocarcinoma using tumor xenografts. Tumor volumes were significantly reduced in the mice treated with Gem + HSV-TK, as compared with the other groups. ${ }^{*} \mathrm{P}<0.05,{ }^{* *} \mathrm{P}<0.01$ vs. the control group. HSV-TK, herpes simplex virus-thymidine kinase; Gem, gemcitabine.

group. The results demonstrated that HSV-TK + GCV suicide gene therapy system can effectively inhibit rat bladder tumor growth. In the present study, HSV-TK gene therapy was administered in combination with gemcitabine to human cholangiocarcinoma QBC939 cell line. As compared with 
the other groups, the in vitro gemcitabine + HSV-TK group exhibited significantly decreased viability. This suggested that combination therapy may have a more potent anti-tumor effect. In order to study the effects of combined HSV-TK gene therapy and gemcitabine in vivo, we developed a mouse model of subcutaneous cholangiocarcinoma was established using tumor xenografts, which confirmed that the combination of HSV-TK and gemcitabine was superior to either HSV-TK or gemcitabine monotherapy. Although gemcitabine immunotherapy temporarily suppressed tumor growth, the tumors relapsed after 7 days. Similarly, HSV-TK monotherapy did not completely suppress tumor growth, demonstrating that HSV-TK/GCV has limited antitumor activity in vivo as a monotherapy. However, the combination of HSV-TK and gemcitabine significantly suppressed the tumors, which confirmed that HSV-TK increases the chemosensitization of the tumors.

Optical imaging was used in the present study to assess tumor growth in individual mice, due to its noninvasive, objective and quantitative features (22). In the present study, QBC939 cells expressing HSV-TK/luciferase were subcutaneously implanted into mice. When injected with luciferin, the tumors emit a visual light signal that can be monitored using a sensitive optical imaging system. As the photon flux emitted from the tumor is proportional to the number of light-emitting cells, this technique can be used to monitor tumor growth and the effect of therapy. In the present study, a decreased photon flux was detected in the murine group treated with HSV-TK + gemcitabine. At days 7 and 14 post-treatment, the mean photon flux value of the murine group administered HSV-TK + gemcitabine significantly decreased, as compared with the other groups. Photon flux values emitted from tumors in the gemcitabine monotherapy groups were decreased, as compared with the HSV-TK-only group. According to our experience, the tumor growth may be monitored using optical imaging for several days before the tumor size becomes palpable or is measurable by US. Furthermore, optical imaging is significantly more sensitive than US in detecting small metastases due to the high signal-to-noise ratio. In the present study, optical imaging successfully detected metastasis in a mouse that US was unable to detect. However, major limitations of optical imaging include its low penetration depth and its inaccuracy at detecting cystic tumors (23). Optical imaging offers high sensitivity for superficially localized lesions, whereas US detects the accurate size of the tumor (24). Therefore, US and optical imaging techniques provided complementary information in the present study.

In conclusion, the results of the present study suggested that gemcitabine is capable of enhancing the therapeutic effects of HSV-TK/GCV in the treatment of cholangiocarcinoma, which can be efficiently monitored by optical imaging and US in vivo. The present study may a novel therapeutic strategy for the management and treatment of cholangiocarcinoma using gemcitabine and HSV-TK/GCV combination therapy.

\section{Acknowledgements}

The present study was supported by the National Natural Science Foundation of China (grant no. 81373714).

\section{References}

1. Nakeeb A, Pitt HA, Sohn TA, Coleman J, Abrams RA, Piantadosi S, Hruban RH, Lillemoe KD, Yeo CJ and Cameron JL: Cholangiocarcinoma. A spectrum of intrahepatic, perihilar and distal tumors. Ann Surg 224: 463-473; discussion 473-465, 1996.

2. Seyama Y and Makuuchi M: Current surgical treatment for bile duct cancer. World J Gastroenterol 13: 1505-1515, 2007.

3. Woo SM, Lee WJ, Kim JH, Kim DH, Han SS, Park SJ, Kim TH, Lee JH, Koh YH and Hong EK: Gemcitabine plus cisplatin versus capecitabine plus cisplatin as first-line chemotherapy for advanced biliary tract cancer: A retrospective cohort study. Chemotherapy 59: 232-238, 2013.

4. Kim MJ, Oh DY, Lee SH, Kim DW, Im SA, Kim TY, Heo DS and Bang YJ: Gemcitabine-based versus fluoropyrimidine-based chemotherapy with or without platinum in unresectable biliary tract cancer: A retrospective study. BMC Cancer 8: 374, 2008.

5. Valle J, Wasan H, Palmer DH, Cunningham D, Anthoney A, Maraveyas A, Madhusudan S, Iveson T, Hughes S, Pereira SP, et al: Cisplatin plus gemcitabine versus gemcitabine for biliary tract cancer. N Engl J Med 362: 1273-1281, 2010.

6. Nagi P, Vickers SM, Davydova J, Adachi Y, Takayama K, BarkerS, Krasnykh V, Curiel DT and Yamamoto M: Development of a therapeutic adenoviral vector for cholangiocarcinoma combining tumor-restricted gene expression and infectivity enhancement. J Gastrointest Surg 7: 364-371, 2003.

7. Andersen JB and Thorgeirsson SS: A perspective on molecular therapy in cholangiocarcinoma: Present status and future directions. Hepat Oncol 1: 143-157, 2014.

8. Pan JG, Luo RQ, Zhou X, Han RF and Zeng GW: Suppression of bladder cancer growth by adeno-associated virus vector-mediated combination of HSV-TK and endostatin in vitro. Clin Lab 59: 1077-1089, 2013.

9. Jarnagin WR, Zager JS, Hezel M, Stanziale SF, Adusumilli PS, Gonen M, Ebright MI, Culliford A, Gusani NJ and Fong Y: Treatment of cholangiocarcinoma with oncolytic herpes simplex virus combined with external beam radiation therapy. Cancer Gene Ther 13: 326-334, 2006.

10. Qu L, Wang Y, Gong L, Zhu J, Gong R and Si J: Suicide gene therapy for hepatocellular carcinoma cells by survivin promoter-driven expression of the herpes simplex virus thymidine kinase gene. Oncol Rep 29: 1435-1440, 2013.

11. Duarte S, Carle G,Faneca H, de Lima MC and Pierrefite-Carle V: Suicide gene therapy in cancer: Where do we stand now? Cancer Lett 324: 160-170, 2012

12. Boucher PD and Shewach DS: In vitro and in vivo enhancement of ganciclovir-mediated bystander cytotoxicity with gemcitabine. Mol Ther 12: 1064-1071, 2005.

13. Wang J, Yang W, Huang Q and Zhai R: Radiosensitization of gemicitabine in human cholangiocarcinoma cell line. Zhonghua Fangshe Zhongliu Xue Za Zhi 17: 123-125, 2008 (In Chinese).

14. Chen L, Guo G, Liu T, Guo L and Zhu R: Radiochemotherapy of hepatocarcinoma via lentivirus-mediated transfer of human sodium iodide symporter gene and herpes simplex virus thymidine kinase gene. Nucl Med Biol 38: 757-763, 2011.

15. Wang J, Yang W, Huang Q, Qian X and Zhai R: Radioenhancement on cholangiocarcinoma by gemcitabine in vivo. Jieru Fang She Xue Za Zhi 10: 685-687, 2007 (In Chinese).

16. Shao D, Li J, Xiao X, Zhang M, Pan Y, Li S, Wang Z, Zhang X, Zheng $H$, Zhang $X$ and Chen L: Real-time visualizing and tracing of HSV-TK/GCV suicide gene therapy by near-infrared fluorescent quantum dots. ACS Appl Mater Interfaces 6: 11082-11090, 2014.

17. Skipworth JR, Keane MG and Pereira SP: Update on the management of cholangiocarcinoma. Dig Dis 32: 570-578, 2014.

18. Valle JW, Furuse J, Jitlal M, Beare S, Mizuno N, Wasan H, Bridgewater J and Okusaka T: Cisplatin and gemcitabine for advanced biliary tract cancer: A meta-analysis of two randomised trials. Ann Oncol 25: 391-398, 2014.

19. Weiss GA, Rossi MR, Khushalani NI, Lo K, Gibbs JF Bharthuar A, Cowell JK and Iyer R: Evaluation of phosphatidylinositol-3-kinase catalytic subunit (PIK3CA) and epidermal growth factor receptor (EGFR) gene mutations in pancreaticobiliary adenocarcinoma. J Gastrointest Oncol 4: 20-29, 2013.

20. Malka D, Cervera P, Foulon S, Trarbach T, de la Fouchardière C, Boucher E, Fartoux L, Faivre S, Blanc JF, Viret F, et al: Gemcitabine and oxaliplatin with or without cetuximab in advanced biliary-tract cancer (BINGO): A randomised, open-label, non-comparative phase 2 trial. Lancet Oncol 15: 819-828, 2014. 
21. Tang W, He Y,Zhou S, Ma Y and Liu G: A novel Bifidobacterium infantis-mediated TK/GCV suicide gene therapy system exhibits antitumor activity in a rat model of bladder cancer. J Exp Clin Cancer Res 28: 155, 2009.

22. Choy G, Choyke P and Libutti SK: Current advances in molecular imaging: Noninvasive in vivo bioluminescent and fluorescent optical imaging in cancer research. Mol Imaging 2: 303-312, 2003.
23. Keereweer S, Van Driel PB, Snoeks TJ, Kerrebijn JD, Baatenburg de Jong RJ, Vahrmeijer AL, Sterenborg HJ and Löwik CW: Optical image-guided cancer surgery: Challenges and limitations. Clin Cancer Res 19: 3745-3754, 2013

24. Keereweer S, Hutteman M, Kerrebijn JD, van de Velde CJ, Vahrmeijer AL and Löwik CW: Translational optical imaging in diagnosis and treatment of cancer. Curr Pharm Biotechnol 3: 498-503, 2012. 